\title{
Usabilidad en aplicaciones de Realidad Virtual Inmersiva Accesible e inclusiva Multi-escenario: Caso práctico
}

\author{
Usability in Accessible and Inclusive Multi-Scenario \\ Immersive Virtual Reality Applications: Case Study
}

\author{
Martha Yaneth Segura Ruiz (ID) Ramiro Osorio Diaz (iD
}

Universitaria Agustiniana, Colombia

OPEN ACCESS

Recibido: 22/09/2021

Aceptado: 22/10/2021

Publicado: 13/12/2021

Correspondencia de autores: martha.segura@uniagustiniana.edu.co

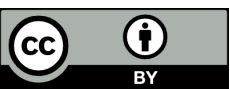

Copyrith 2020

by Investigación e Innovación en Ingenierías
Resumen

Objetivo: Evaluar un producto de software, resultado del proyecto de investigación titulado Metodología para el desarrollo de aplicaciones de Realidad Virtual Inmersiva que cumpla con los principios del diseño universal, para validar la inclusión y accesibilidad en la experiencia de usuarios con diferentes capacidades. Metodología: La realidad virtual es una tecnología que permite al usuario simular experiencias en un mundo virtual en 3D creado por el hombre mediante software y que requiere de un dispositivo adicional como gafas o casco. Sin embargo, su utilización está limitada para personas que presentan algún tipo de discapacidad. El proceso de desarrollo de aplicaciones de realidad virtual accesibles está obligado a incluir a todas las personas. La metodología RVI-A aplicada se fundamenta en los requisitos de accesibilidad para personas con discapacidad visual y auditiva. Resultados: Para evaluar la calidad del software desde la óptica de los usuarios que presentan discapacidades auditivas y visuales se realizaron pruebas de usabilidad centradas en el usuario, mediante métodos heurísticos con expertos, y empíricos con usuarios reales, para determinar si el software cumplía con los criterios de entendimiento, aprendizaje, operabilidad, atracción y conformidad de uso. Conclusiones: El trabajo permitió concluir mediante los resultados de usabilidad que el software cumplió los requisitos del usuario y la calidad esperada, mientras que la experiencia de usuario fue positiva, logrando incluir a personas con discapacidad visual y auditiva, disminuyendo la brecha tecnológica que existe entre el mundo real y el mundo virtual, mejorando este tipo de aplicaciones.

Palabras clave: Realidad Virtual, accesibilidad, inclusión, usabilidad, heurística, pruebas

Abstract

Objective: To evaluate a software product, the result of the research project entitled Methodology for the development of immersive virtual reality applications that comply with the principles of universal design, in order to validate the inclusion and accessibility of the experience for users with different abilities. Methodology: Virtual reality is a technology that allows the user to simulate experiences in a 3D virtual world created by humans through software and that requires an additional device such as glasses or a helmet. However, its use is limited for people with disabilities. The process of developing accessible virtual reality applications is bound to include all people. The applied RVI-A methodology is based on the accessibility requirements for visually and hearing impaired people. Results: To assess the quality of the software from the perspective of users with hearing and visual disabilities, user-centred usability tests were conducted using heuristic methods with experts, and empirical methods with real users, to determine whether the software met the criteria of understanding, learning, operability, attractiveness and conformity of use. Conclusions: The work allowed us to conclude through the usability results that the software met the user requirements and the expected quality, while the user experience was positive, managing to include visually and hearing impaired people, decreasing the technological gap that exists between the real world and the virtual world, improving this type of applications.

Keywords: Virtual reality, accessibility, inclusion, usability, heuristic, testing.

Como citar (IEEE): M. Segura-Ruiz., y R. Osorio Diaz. "Usabilidad en aplicaciones de Realidad Virtual Inmersiva Accesible e inclusiva Multi-escenario: Caso práctico". Investigación e Innovación en Ingenierías, vol. 9, n³, 82-92, 2021. DOI: https://doi.org/10.17081/ invinno.9.3.5563 


\section{Introducción}

El desarrollo de aplicaciones de realidad virtual inmersiva se ha generalizado en los últimos años en diferentes áreas del conocimiento, es una tecnología que tiene características específicas como la interactividad en tiempo real, permitiendo al usuario experimentar en el entorno virtual 3D, esto contribuye a que el usuario sienta que se encuentra inmerso en dicha realidad, una realidad paralela.

Para realizar la inmersión en aplicaciones de realidad virtual se deben tener en cuenta los canales sensoriales del ser humano, en especial la vista y el oído, por lo que personas que presentan algún tipo de discapacidad sensorial no pueden acceder y utilizar el software, es decir, que este tipo de software en su gran mayoría no es accesible ni inclusivo.

En una primera fase, el proyecto de investigación titulado Metodología para el desarrollo de aplicaciones de Realidad Virtual Inmersiva VR que cumpla con los principios del diseño universal, desarrollado durante el año 2018, adaptó una metodología a partir del análisis de los diferentes métodos de desarrollo ágiles y como consecuencia se realizó un prototipo funcional, buscando resolver el problema de accesibilidad en entornos de realidad virtual inmersiva, aplicando empíricamente la metodología.

En una segunda fase del proyecto de investigación se analizaron las necesidades de las personas con discapacidad sensorial y personas mayores, se determinaron los requisitos funcionales y no funcionales, basados en información del Instituto Nacional para Sordos (INSOR) y el Instituto Nacional para Ciegos (INCI) de Colombia. Se definió una arquitectura de software, para culminar con un prediseño de la aplicación.

En la tercera fase a partir del prototipo diseñado se construyó la aplicación de realidad virtual inmersiva accesible para todas las personas incluidas aquellas que presentaran algún grado de discapacidad visual o auditiva; se adicionó un módulo sobre protocolos de bioseguridad frente al Covid-19, y un framework para editar objetos 2D y 3D, con el fin de personalizar la aplicación de acuerdo a las necesidades del usuario. El resultado es un aporte a la inclusión de personas con discapacidad sensorial auditiva y visual, logrando derribar barreras de acceso en el uso de software de este tipo.

El proyecto fortaleció la línea de investigación en Estudios en desarrollo de software y en el área estratégica Aplicación de buenas prácticas de desarrollo del software, aportando a los estudiantes, egresados y docentes del Programa de Tecnología en Desarrollo de Software de Uniagustiniana nuevos conocimientos en la construcción de software accesible de realidad virtual inmersiva, creando un marco de referencia para proyectos similares y generando un proceso de evaluación para asegurar la usabilidad del mismo.

El documento explica el proceso de evaluación del software resultado del proceso de investigación y desarrollo tecnológico, expone el análisis de las pruebas de usabilidad y heurísticas realizadas para el refinamiento del software en un caso práctico, teniendo como actor principal al usuario con diversas capacidades.

\section{Metodología}

Los trabajos previos sobre usabilidad en aplicaciones de realidad virtual [1] y el diseño del entorno virtual [2] se han centrado en simulaciones y evaluaciones heurísticas. Las aplicaciones modernas en realidad virtual también se han enfocado en la aplicación de patrones de diseño [3].

A nivel de desarrollos tecnológicos, plataformas de inclusión [4], reflejan la importancia del diseño universal. Los recorridos virtuales [5] usados para publicidad de espacios e inmersivos [6] permiten a los 
usuarios tener experiencias sensoriales satisfactorias que se aplican en diferentes espacios.

El estudio y desarrollo de interfaces sensitivas de realidad virtual y aumentada para invidentes en dispositivos portátiles [7] prueba que usuarios con limitaciones visuales también pueden acceder a este tipo de tecnologías. Los frameworks para crear entornos en 3D han sido de utilidad para investigadores, científicos, funcionarios y público, como es el caso de "Geospatial VR" [8], desarrollado en el motor Unity.

Para la Organización Internacional de Estandarización (ISO), norma ISO/IEC 9126, "La usabilidad se refiere a la capacidad de un software de ser comprendido, aprendido, usado y ser atractivo para el usuario, en condiciones específicas de uso", el desarrollo de la aplicación se enfocó a cumplir con estos criterios.

El grado de usabilidad [9] de un sistema se puede definir como una medida empírica y relativa de la usabilidad del mismo: Empírica, porque no se basa en opiniones o sensaciones sino en pruebas de usabilidad, realizadas en laboratorio. Relativa, porque el resultado no es ni bueno ni malo, sino que depende de las metas planteadas o de una comparación con otros sistemas similares.

\section{Metodología RVI-A}

La construcción de la aplicación a partir de la Metodología para el desarrollo de aplicaciones de realidad virtual Inmersiva que cumpla los principios del diseño universal [10], se convirtió en un marco de referencia, que simplificó las tareas de los desarrolladores de aplicaciones de este tipo, ya que con conocimientos previos pueden integrar contenido multimedia, modelado 3D y scripts, sirviendo para proyectos más complejos y de mayor tamaño.

La metodología adaptada profundiza en la elicitación de requisitos funcionales y no funcionales basados en el diseño universal necesarios para la codificación de aplicaciones de Realidad Virtual Inmersiva Accesible e Inclusiva RVI-A. Esta metodología es de gran utilidad y mejora aspectos en la construcción de software accesible, se fundamenta en procesos iterativos e incrementales, y pone al usuario como eje en la evaluación del software. En la Figura 1 se observan las cuatro fases y subfases de la metodología.

Figura 1. Fases metodología para el desarrollo de aplicaciones de Realidad Virtual Inmersiva RVI-A que cumpla los principios del diseño universal.

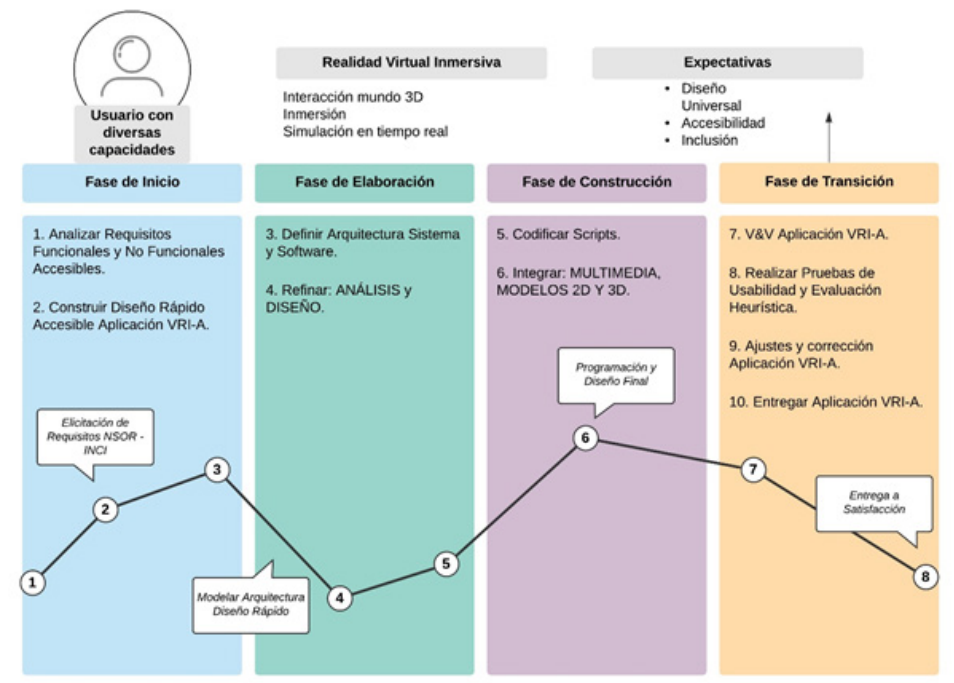

Fuente: elaboración propia. 
Se construyó la aplicación en realidad virtual inmersiva que cuenta con accesibilidad para personas con discapacidad visual mediante sonidos inmersivos, audio descripciones, contrastes, manejo de colores, y discapacidad auditiva con lengua de señas, imágenes de excelente calidad, contrastes y close caption. Debido a la pandemia del año 2020 se incluyó al software un módulo de protocolos de bioseguridad frente al Covid-19.

Otra funcionalidad del software es el editor de tours VR Creator para personalizar en cualquier escenario la creación de recorridos en realidad virtual inmersiva permitiendo a los usuarios diseñar, construir y exhibir lo realizado, agregar objetos 3D para personalizar el tour por el usuario. Se puede ejecutar desde dispositivos Android 7.0 o computadoras con sistema operativo Windows 10.

Se diseñó una estructura modular fundamentada en la arquitectura de microservicios que permite integrar distintos servicios que pueden actuar de forma independiente. Existen módulos base como el cambio de escena, las opciones de configuración y otros no obligatorios como el módulo de Covid-19. Los módulos Blind VR y VR Creator dependen de la utilidad que le presente al usuario. Se pueden activar o desactivar opciones, estas decisiones se toman a partir de la definición de requisitos.

Para hacer uso de la función importar objeto 3D es necesario acatar los formatos permitidos por la aplicación preferiblemente utilizar el renderizado en OBJ para evitar inconvenientes en la carga y escala del objeto en cuestión esto permitirá al usuario tener una agradable experiencia de uso.

En la Figura 2 se muestran los módulos de la aplicación: Tour VR, Covid VR, Blind VR, el módulo para personas con discapacidad visual, y VR Creator, framework para personalizar los escenarios 3D y objetos 2D. También se destacan en el menú principal las opciones de personalización para personas con discapacidad auditiva (señas, subtítulos, sonidos inmersivos). Cabe destacar que durante el recorrido del Tour VR los usuarios encuentran vídeos con lengua de señas que traducen la información mostrada. La aplicación soporta varios lenguajes.

Figura 2. Menú principal de la aplicación RVI-A.

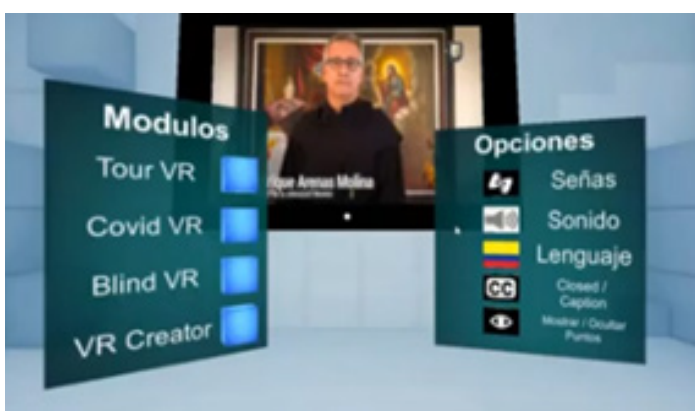

Fuente: elaboración propia.

Durante el diseño de la aplicación se realizó un plano de las áreas de la Universitaria Agustiniana donde las personas con discapacidad visual podían interactuar a través de sonidos inmersivos, y con indicaciones específicas identificar su ubicación, el plano se muestra en la Figura 3. 
Figura 3. Interfaz del módulo Blind VR.

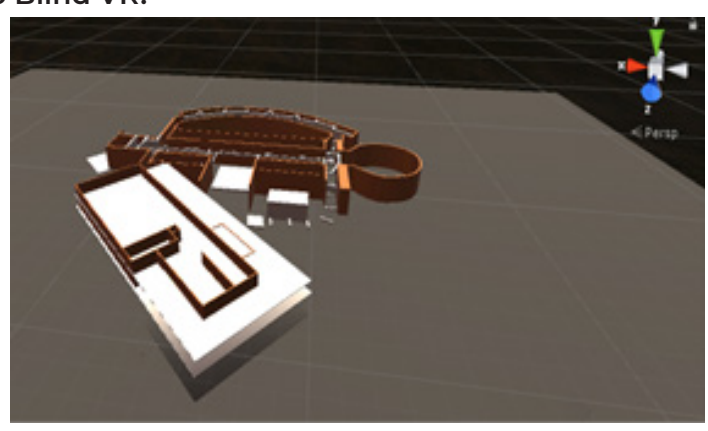

Fuente: elaboración propia.

Para el uso de la aplicación por parte de las personas con discapacidad visual, se desarrolló un script que configuraba un control de mano a través de teclas con los que el usuario puede interactuar con la aplicación, como se puede observar en la Figura 4.

Figura 4. Interfaz del mando en Blind VR.

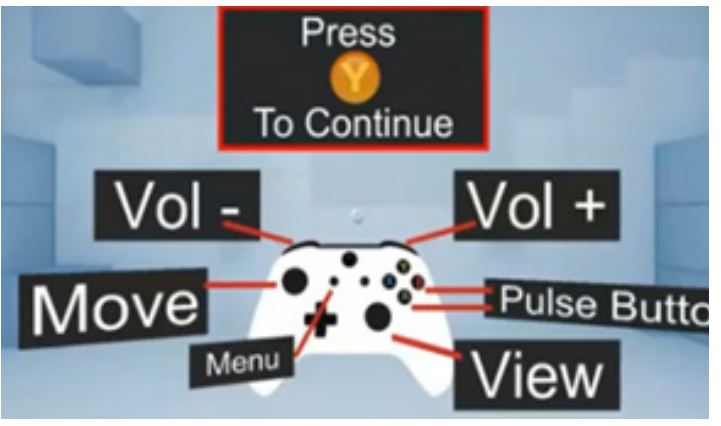

Fuente: elaboración propia.

Las personas que no presentan ningún tipo de discapacidad pueden realizar un recorrido controlado por las diferentes instalaciones, en la Figura 5 se muestra la escena que recrea un laboratorio de computadores.

Figura 5. Interfaz del módulo Tour VR.

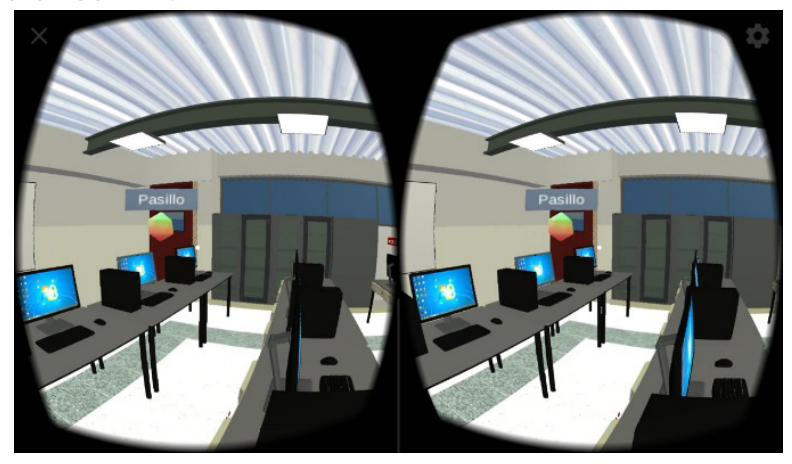

Fuente: elaboración propia.

Con el fin de personalizar la aplicación se adiciona un framework para el desarrollo dinámico en cualquier entorno de realidad virtual inmersiva (multi-escenario). La interfaz del framework se presenta en la Figura 6. 
Figura 6. Interfaz del módulo VR Creator.

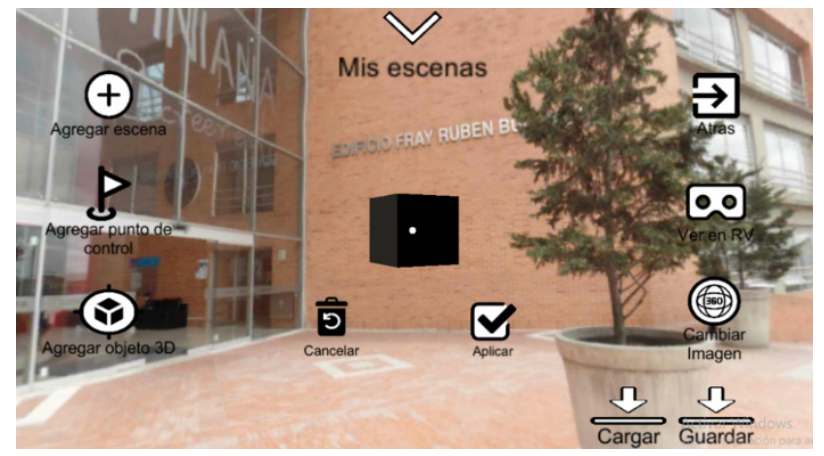

Fuente: elaboración propia.

Para apropiar a funcionarios y visitantes de la Universitaria Agustiniana sobre los protocolos de bioseguridad frente a la Covid-19, se incluyó un módulo que indica a las personas sobre el autocuidado para evitar la propagación del nuevo virus. Desde el ingreso a la institución se presentan carteles con información específica como se muestra en la Figura 7.

Figura 7. Interfaz entrada módulo Tour VR.

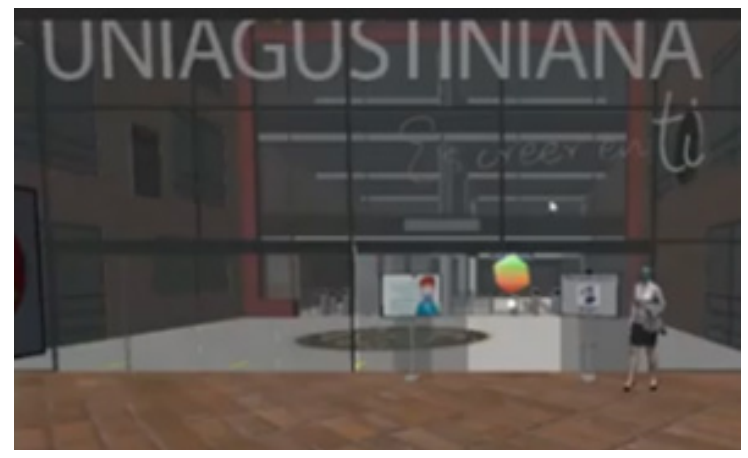

Fuente: elaboración propia.

La aplicación tiene como base el motor de videojuegos Unity para el desarrollo del entorno virtual, con el lenguaje de programación multiparadigma C\# (c sharp), que permitió implementar la aplicación RVI-A. El modelado 3D de la aplicación se construyó en Autodesk 3ds Max que es un programa de creación de gráficos y animación 3D.

Los archivos del software quedan guardados en un apartado que se genera dentro del Apk, que se debe instalar en un celular que cuente con giroscopio y con soporte de realidad virtual. Finalmente, la aplicación se utiliza con cualquier tipo de visor de realidad virtual, no se requiere de una conexión a internet para su funcionamiento.

La aplicación en realidad virtual inmersiva es diferente a otro tipo de aplicaciones, para ejecutarla, los usuarios necesitaban un visor con un celular. Para desplazarse por la aplicación, se utilizan los movimientos de la cabeza y ojos, en el caso de personas con discapacidad auditiva, las imágenes y los videos de señas aportan a su inmersión, y para las personas con discapacidad visual, el canal de interacción es el oído, su interacción se realiza a través de sonidos inmersivos, señales auditivas y audio descripciones. 
El equipo del proyecto de investigación estuvo conformado por docentes, egresados y estudiantes del semillero permeando el currículo del programa de Tecnología en Desarrollo de Software de la Universitaria Agustiniana.

\section{Pruebas para asegurar la calidad del software}

Durante la fase de transición se realizaron procesos de validación y verificación de la aplicación, donde se revisó el cumplimiento de cada uno de los requisitos funcionales y no funcionales por parte del equipo de desarrollo, y se comprobó la correcta funcionalidad del sistema hardware (celular, visor RV) y software (aplicación VRI-A).

Las pruebas de usabilidad y la evaluación heurística se llevaron a cabo en dispositivos que cumplían las especificaciones técnicas definidas en el manual de usuario, fueron aplicadas a usuarios reales con diversas capacidades y a desarrolladores expertos en programación de aplicaciones con Unity.

La fase de pruebas fue sumamente importante ya que en ella se comprobó el funcionamiento de la aplicación, durante este proceso se detectaron errores derivados de la etapa de programación, que se resolvieron satisfactoriamente, ya que, tratándose de un proceso iterativo, el proceso de refinación de requerimientos y requisitos se repitió cierto número de veces, siempre con el acompañamiento del usuario final.

Durante la primera fase se analizaron las heurísticas que se podían aplicar para medir el nivel de usabilidad de este tipo de aplicaciones, debido a que estas no pueden medir todas las características de la realidad virtual. Las heurísticas priorizadas se basaron en los principios de Nielsen [11], adaptado a mundos virtuales [12] y realidad virtual.

Durante la segunda fase se elaboró un banco de preguntas, priorizando las siguientes heurísticas: Claridad; simplicidad; retroalimentación; coherencia; baja carga de memoria; flexibilidad y eficacia de uso; orientación y navegación; control de las gafas; visualización; personalización del menú; interacción con el mundo; ley de la física; comunicación; prevención de errores; ayudar a los usuarios a recuperarse de los errores; ayuda y documentación.

Para las pruebas de usabilidad se determinó un grupo de preguntas con el equipo de investigación y desarrollo, orientadas a los criterios de entendimiento, aprendizaje, operabilidad, atracción y conformidad de uso.

Los expertos contestaron las preguntas paralelamente utilizaban la aplicación. Los usuarios finales probaron el software en sus celulares, con un visor de realidad virtual, y un mando de control, posterior a su experiencia inmersiva con la aplicación contestaron las preguntas de usabilidad formuladas previamente.

\section{Resultados}

Los resultados de las pruebas de usabilidad y la evaluación heurística demuestran que es necesario realizar más experimentos para el perfeccionamiento de la evaluación de este dominio de aplicación especifico, en realidad virtual inmersiva accesible e inclusivo.

La evaluación heurística realizada por los ingenieros y desarrolladores de software se enfocó en analizar la aplicación desde la óptica y el sentir de los usuarios, lo que determinó unos ajustes y correcciones a la aplicación, para continuar con las pruebas de usuario final. La evaluación fue realizada por (22) ingenieros y 
desarrolladores de software con más de dos años de experiencia en programación con Unity, y conocimiento en creación de aplicaciones de realidad virtual inmersiva.

En la siguiente tabla se muestra la información relacionada con las heurísticas, el total de preguntas, su cumplimiento y el número de problemas detectados.

Tabla 1. Heurísticas priorizadas en la evaluación.

\begin{tabular}{|c|c|c|c|c|c|}
\hline No & Heuristica & $\begin{array}{l}\text { Número total } \\
\text { preguntas }\end{array}$ & Si cumple & No cumple & $\begin{array}{c}\text { Cantidad } \\
\text { Problemas } \\
\text { Detectados- } \\
\text { Corregidos }\end{array}$ \\
\hline 1 & Claridad & 11 & $86,4 \%$ & $13,6 \%$ & 2 \\
\hline 2 & Simplicidad & 4 & $86,4 \%$ & $13,6 \%$ & 0 \\
\hline 3 & Retroalimentación & 5 & $86,4 \%$ & $13,6 \%$ & 1 \\
\hline 4 & Coherencia & 5 & $81,8 \%$ & $18,2 \%$ & 2 \\
\hline 5 & Baja carga de memoria & 3 & $81,8 \%$ & $18,2 \%$ & 0 \\
\hline 6 & Flexibilidad y eficacia de uso & 2 & $90,9 \%$ & $9,1 \%$ & 1 \\
\hline 7 & Orientación y navegación & 5 & $81,8 \%$ & $18,2 \%$ & 3 \\
\hline 8 & Control de las gafas & 2 & $86,4 \%$ & $13,6 \%$ & 1 \\
\hline 9 & Visualización & 4 & $90,9 \%$ & $9,1 \%$ & 2 \\
\hline 10 & Personalización del menú & 4 & $86,4 \%$ & $13,6 \%$ & 1 \\
\hline 11 & Interacción con el mundo & 2 & $81,8 \%$ & $18,2 \%$ & 2 \\
\hline 12 & Ley de la física & 2 & $90,9 \%$ & $9,1 \%$ & 1 \\
\hline 13 & Comunicación & 4 & $95,5 \%$ & $4,5 \%$ & 1 \\
\hline 14 & Prevención de errores & 3 & $81,8 \%$ & $18,2 \%$ & 2 \\
\hline 15 & $\begin{array}{l}\text { Ayudar a los usuarios a } \\
\text { recuperarse de los errores }\end{array}$ & 3 & $81,8 \%$ & $18,2 \%$ & 1 \\
\hline \multirow[t]{2}{*}{16} & Ayuda y documentación & 4 & $81,8 \%$ & $18,2 \%$ & 2 \\
\hline & Total Heurísticas priorizadas & 63 & $85,8 \%$ & $14,2 \%$ & 22 \\
\hline
\end{tabular}

\section{Fuente. Elaboración propia.}

Los evaluadores identificaron un total de veintidós (22) problemas, que fueron solucionados para garantizar la calidad de la aplicación, dentro de los cuales estaban: el rediseño del menú, mejora en la interacción con las estructuras para las personas con discapacidad visual, un incremento y mejora en los sonidos inmersivos y audios, la optimización en la carga de los videos de lengua de señas, entre otros. En general, el resultado de la evaluación heurística arrojó un cumplimiento del $85,8 \%$ de la aplicación por parte de los expertos.

Las pruebas de usabilidad se dirigieron a una muestra de diecisiete (17) personas con diferentes capacidades, edades, conocimientos y experiencias, personas con discapacidades auditivas y visuales, se trató en lo posible que las personas encuestadas encajaran con el perfil de usuario. La organización de las pruebas según los criterios de usabilidad, número de preguntas, su cumplimiento, la cantidad de problemas detectados, se muestra en la siguiente tabla. 
Tabla 2. Criterios de usabilidad evaluados.

\begin{tabular}{|cccccc} 
No & Criterio de Usabilidad & $\begin{array}{c}\text { Número } \\
\text { total } \\
\text { preguntas }\end{array}$ & $\begin{array}{c}\text { Si } \\
\text { cumple }\end{array}$ & No cumple & $\begin{array}{c}\text { Cantidad Problemas } \\
\text { Detectados-Corregidos }\end{array}$ \\
\hline U1 & Entendimiento & 6 & $82,4 \%$ & $17,6 \%$ & 2 \\
\hline U2 & Aprendizaje & 4 & $88,2 \%$ & $11,8 \%$ & 3 \\
\hline U3 & Operabilidad & 7 & $94,1 \%$ & $5,9 \%$ & 2 \\
\hline U4 & Atracción & 5 & $88,2 \%$ & $11,8 \%$ & 4 \\
\hline U5 & Conformidad de uso & 8 & $94,1 \%$ & $5,9 \%$ & 0 \\
\hline & Total Criterios de Usabilidad & 30 & $88,2 \%$ & $11,8 \%$ & 11 \\
\hline
\end{tabular}

\section{Fuente. Elaboración propia.}

Como resultado de las pruebas, se observó que el $88,2 \%$ de las personas que realizaron la evaluación de usabilidad consideraron que se cumplían los criterios analizados. Los problemas detectados en total fueron once (11), dentro de los cuales se encontró que el uso de la aplicación ocasionaba mareos y fatiga visual. Los problemas específicos de diseño y desarrollo fueron corregidos totalmente.

El análisis de las pruebas de usabilidad determinó que la aplicación cumplía con las expectativas de usabilidad logrando un $94.1 \%$ de operabilidad para las funcionalidades y conformidad de uso, 82,4\% para el entendimiento, $88,2 \%$ en aprendizaje y atracción. Las pruebas también sirvieron para identificar qué mejoras serían óptimas para una futura versión de la aplicación.

Para el módulo Blind VR, se realizó una prueba de usabilidad específica a cinco (5) personas con baja visión y ciegas. Durante la prueba se ejecutó el módulo, la interacción se realizaba por medio del giroscopio del celular y un mando de video juegos. Después de realizar el recorrido por parte del usuario se aplicaba la encuesta. Para esta encuesta se utilizó una escala de 1 a 5, siendo 1-muy regular, 2-regular, 3-bueno, 4-muy bueno y 5 -excelente. Los resultados determinaron que los audios y sonidos inmersivos permitieron guiar al usuario durante el recorrido, aunque se demoraba en ubicarse dentro de la aplicación, y en aprender a utilizar el control de mando. En la Figura 8 se observa el puntaje final otorgado por las personas con discapacidad visual que evaluaron el software.

Figura 8. Evaluación final por personas con discapacidad visual.

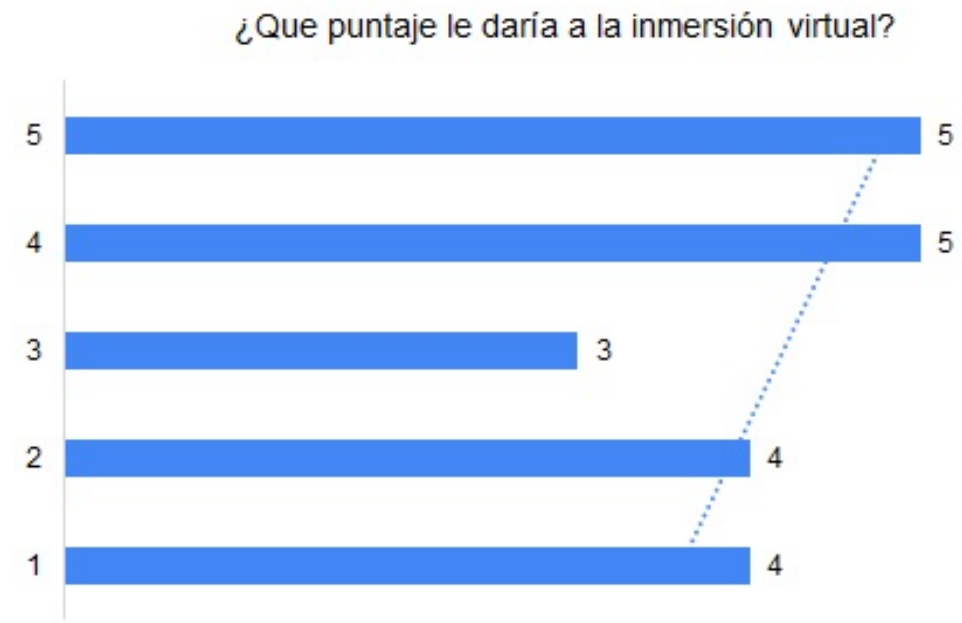

Fuente. Elaboración propia. 
Estos resultados muestran que la evaluación del software de realidad virtual inmersiva es un proceso de retroalimentación permanente que coadyuva a una mejor experiencia del usuario con diversas capacidades.

\section{Conclusiones}

Se cumplieron los objetivos del proyecto de investigación, de la aplicación VRI-A y de las pruebas de usabilidad, evaluando la experiencia del usuario según los criterios de entendimiento, aprendizaje, operabilidad, atracción y conformidad de uso. Se presentó cierta dificultad en su realización por las restricciones de movilidad ocasionadas por la pandemia, en cuanto al número de participantes.

Las evaluaciones heurísticas realizadas por expertos permitieron realizar cambios necesarios en la aplicación para asegurar su calidad antes de realizar las pruebas de usabilidad con usuarios reales. Mostrando cada uno de los problemas de la aplicación con alto grado de claridad.

Las pruebas realizadas en la aplicación dieron resultados positivos para comprobar la validez de la metodología propuesta en la investigación, generando buenas prácticas en el ciclo de desarrollo de software accesible. La mejora de la calidad de los procesos de desarrollo de software de realidad virtual inmersiva, junto con el aumento de la capacidad de estudiantes y profesores para interactuar con las nuevas herramientas, tiene el potencial de transformar significativamente este tipo de aplicaciones centradas en el diseño para todos.

Los usuarios con discapacidad visual y auditiva mostraron altos niveles de aceptación y satisfacción por su inclusión en el proceso de desarrollo de la aplicación y cumplieron sus expectativas en cuanto a las experiencias sensoriales que fueron proporcionadas.

Se espera que el software evolucione en el tiempo con los nuevos requerimientos de usuario, herramientas de desarrollo y hardware especializado que soporte la Realidad Virtual Inmersiva Accesible RVI-A. Es necesario consolidar un grupo de heurísticas de usabilidad para el dominio de las aplicaciones de realidad virtual inmersiva accesible en futuras investigaciones.

\section{Referencias bibliográficas}

1. W. Wang, J. Guo \& J. Cheng, "Usability of Virtual Reality Application Through the Lens of the User Community: A Case Study", en Extended Abstracts of the 2019 CHI Conference on Human Factors in Computing Systems, pp. 1-6. DOI: https://doi.org/10.1145/3290607.3312816

2. A. Sutcliffe, C. Poullis, A. Gregoriades, I. Katsouri, A. Tzanavari, \& K. Herakleous, "Reflecting on the Design Process for Virtual Reality Applications", International Journal of Human-Computer Interaction, vol. 35, no. 2, pp. 168-179, 2019. DOI: https://doi.org/10.1080/10447318.2018.1443898

3. R. Mosquera, “Diseño de Experimento: Aproximación metodología para el desarrollo de una aplicación interactiva de realidad virtual Inmersiva, para recrear la terapia espejo en la rehabilitación de pacientes amputados de miembros inferiores con dolor de miembro fantasma", en 4to Congreso Internacional AmITIC 2017, Popayán, Colombia. 6 al 8 de septiembre de 2017, pp. 224-228. Disponible en: https://core.ac.uk/reader/234021146 
4. G. Lafuente, C. Ballesteros \& J. Filippi, “Desarrollo de una plataforma de capacitación a distancia utilizable y abierta para personas con discapacidad visual", en XX Workshop de Investigadores en Ciencias de la Computación (WICC 2018, Universidad Nacional del Nordeste), 26 y 27 de abril de 2018, pp. 1227-1231.

5. Y. Olvera \& M. Gea, “Desarrollo de realidad Virtual para publicitar el Panteón Inglés ubicado en Real del Monte Hidalgo". Programación Matemática y Software, vol. 9, no. 3, pp. 44-52, 2017. Disponible en: http://www.progmat.uaem.mx:8080/Vol9num3/vol9num3art6.pdf

6. M. Elfarargy, \& A. Rizq. VIR MUF: The Virtual Museum Framework. Scalable Computing: Practice and Experience, vol. 19, no. 2, pp. 175-180, 2018. DOI: https://doi.org/10.12694/scpe.v19i2.1349

7. N. Paredes, "Estudio y desarrollo de interfaces sensitivas de realidad virtual y aumentada para invidentes en dispositivos portátiles", Tesis Doctoral, Universidad Politécnica de Madrid, 2018.

8. Y. Sermet, \& I. Demir, I. "Geospatial VR: A Web-basedVirtual Reality Framework for Collaborative Environmental Simulations". pp. 2, 2020. DOI: https://doi.org/10.31223/osf.io/ks2yz

9. W. Sánchez, "La usabilidad en Ingeniería de Software: definición y características," Revista IngNovación, no. 2, pp. 7-22, 2011. Disponible en: http://hdl.handle.net/11715/519

10. M.Segura \& R. Fonseca, "Methodology for the development of VR immersive virtual reality applications that comply with universal design principles. Case study: Uniagustiniana immersive virtual tour", en Proceedings de JIISIC 2018 - Jornadas Iberoamericanas de Ingeniería de Software e Ingeniería del Conocimiento, pp. 27-36, 2018.

11. J. Nielsen. 10 Heuristics for User Interface Design. https://www.nngroup.com/articles/ten-usabilityheuristics/, 1995.

12. Rusu, C., Muñoz, R., Roncagliolo, S., Rudloff, S., Rusu, V., \& Figueroa, A, “Usability heuristics for virtual worlds", en Proceedings of the Third International Conference on Advances in Future Internet, pp. 16-19, 2011. 